\title{
CONVECTION-DIFFUSION EQUATIONS WITH RANDOM INITIAL CONDITIONS
}

\author{
MIŁOSZ KRUPSKI \\ Uniwersytet Wroctawski, Instytut Matematyczny \\ pl. Grunwaldzki 2/4, 50-384 Wroctaw
}

\begin{abstract}
We consider an evolution equation generalising the viscous Burgers equation supplemented by an initial condition which is a homogeneous random field. We develop a non-linear framework enabling us to show the existence and regularity of solutions as well as their long time behaviour.
\end{abstract}

\section{INTRODUCTION}

The main objective of this paper is to investigate solutions to a non-local analogue of the viscous Burgers equation with random initial conditions

$$
\left\{\begin{array}{lr}
\partial_{t} u+(-\Delta)^{s} u=\nabla_{z} f(u) & \text { on }[0, \infty) \times \mathbb{R}^{d}, \\
u(0) \stackrel{d}{=} u_{0} & \text { on } \mathbb{R}^{d} .
\end{array}\right.
$$

Here the operator $-(-\Delta)^{s}$ denotes the (now) standard fractional Laplace operator with $s \in\left(\frac{1}{2}, 1\right]$ and the initial condition $u_{0}$ is a real, homogeneous, isotropic random field (as defined in Section 2) whose finite-order moments are all bounded. By $\nabla_{z}$ we denote the directional derivative. The function $f$ is a smooth function of polynomial growth.

Such equations have been studied thoroughly in the deterministic case. In papers BKW99, BKW01a, BKW01b the authors consider initial conditions which are integrable functions and describe certain properties of solutions that resonate with some of the results presented here. However, in the context of random initial data new methods had to be developed. Important results were obtained for bounded (deterministic) initial conditions DGV03 and we rely on them where possible (see Lemmas 5.6, 5.7). Interesting developments were recently described in [S17.

In general, partial differential equations with random initial data (homogeneous, stationary, isotropic, etc.) have been examined before, notably to describe certain physical phenomena, such as the Large Scale Structure of the Universe [AMS94, Woy98, JW01.

More specifically, there are numerous results available on the local Burgers equation (i.e. equation (1) with $s=1$ and $f(u)=u^{2}$ ) or very similar equations with random initial data [AL01, AL02, Bak01, Leo99, LW98. Equations of this type, however, have a curious property, exploited by the Hopf-Cole transformation, allowing them to be treated essentially as linear problems. Such a reduction is not known to be possible both in the non-local setting (i.e. with the fractional Laplacian) and for a more general function $f$, which forces us to conduct a more involved non-linear analysis of the problem.

E-mail address: milosz.krupski@math.uni.wroc.pl. 
A significant part of this analysis are a priori estimates, in the context of random initial data. Early results were already obtained by Rosenblatt [Ros68] (in the local setting). Taking them as an inspiration we obtained new and much more general estimates. In fact, in Theorem 5.10 we prove that for a solution $u(t)$ to problem (1) we have $E|u(t)|^{p} \leq E\left|u_{0}\right|^{p}$ for every $t \geq 0$.

One difficulty when working with random fields is the question of regularity of its individual realisations. As it turns out, it is impossible to directly apply the classical theory "pathwise", treating $x \mapsto u_{0}(x, \omega)$ as an initial condition of the non-random problem for every $\omega \in \Omega$ separately (cf. Proposition 3.8 and Remark 3.9).

On the other hand, restricting the problem to admit only homogeneous random fields has a technical advantage. By calculating the expected value $E$ not only we "eliminate" the variable $\omega$, as is normally the case, but also $x$ (because the field has identical distribution at every point in space the result is constant, see Remark 2.3). However simple, this property is essential to obtain e.g. the analogue of the Stroock-Varopoulos inequality (see [VSCC92, LS96) in Lemma 5.9. Another important observation is expressed by Lemma 2.11 and we invite the readers to turn their attention to it.

The remainder of the paper is structured as follows. After introducing the notation and basic concepts in Section 2, in Section 3 we review the results on the linear equation, which is the starting point for the rest of the theory. In Section 4 we construct solutions in the case when the function $f$ is assumed to be Lipschitz. In Section 5 we are able to establish the a priori estimates. Finally, in Section 6 we study the problem for functions $f$ of polynomial growth. The main result on the existence of solutions to problem (11) is expressed in Theorem 6.5

This paper is a continuation of previous work Kru17 which dealt with linear problems.

\section{ISOMETRY-INVARIANT RANDOM FIELDS}

2.1. Basic notation. We denote the Borel sigma-algebra on $\mathbb{R}^{d}$ by $\operatorname{Bor}\left(\mathbb{R}^{d}\right)$ and the Lebesgue measure by $d x$. We use the Fourier transform defined as

$$
\mathcal{F}\{f\}(\xi)=\int_{\mathbb{R}^{d}} e^{i \xi \cdot x} f(x) d x .
$$

Given a measure space $(X, \Theta, \mu)$, by $L^{p}(X, \Theta, \mu)$ we denote the space of all $\Theta$ measurable real functions such that the norm defined in the case $1 \leq p<\infty$ as

$$
\|f(x)\|_{L^{p}(X, \Theta, \mu)}^{p}=\int_{X}|f|^{p} d \mu=\int_{X}|f(x)|^{p} \mu(d x),
$$

or in the case of $p=\infty$ as the value

$$
\|f(x)\|_{L^{\infty}(X, \Theta, \mu)}=\operatorname{esssup}_{x \in X}|f|=\inf \{y \in X: \mu\{x \in X:|f(x)|>y\}=0\},
$$

is finite. Usually we shorten the notation and write $L^{p}(X, \mu)$.

Let $\left(X,\|\cdot\|_{X}\right)$ be a subset of a normed space. For every fixed $K \geq 0$ we may define the Bielecki norm

$$
\|u \mid\|_{K, X}=\sup _{t \geq 0} e^{-t K}\|u(t)\|_{X}
$$

and the space

$$
\mathcal{B}_{K, X}=\left\{u \in C([0, \infty), X):\|u\|_{K, X}<\infty\right\} .
$$

Let us fix a probability space $(\Omega, \Sigma, P)$ and denote $L^{p}(\Omega)=L^{p}(\Omega, \Sigma, P)$. The particular case of $p=2$ constitutes a Hilbert space with the standard inner product 
$E X Y$ defined for all $X, Y \in L^{2}(\Omega)$. We write

$$
\left(X_{1}, \ldots, X_{k}\right) \stackrel{d}{=}\left(Y_{1}, \ldots, Y_{k}\right), \quad \text { where } X_{i}, Y_{i} \in L^{p}(\Omega) \text { and } k \in \mathbb{N}
$$

if both random vectors have the same probability distributions.

2.2. Random fields. Consider $L^{p}(\Omega)$-continuous and $L^{p}(\Omega)$-bounded random field $u$ on $\mathbb{R}^{d}$, i.e. $u \in C_{b}\left(\mathbb{R}^{d}, L^{p}(\Omega)\right)$. We endow this space with the norm

$$
\|u\|_{p}=\sup _{x \in \mathbb{R}^{d}}\|u(x)\|_{L^{p}(\Omega)}
$$

For $u, v \in C_{b}\left(\mathbb{R}^{d}, L^{p}(\Omega)\right)$ we say that $u \stackrel{d}{=} v$ if

$$
\left(u\left(x_{1}\right), \ldots, u\left(x_{n}\right)\right) \stackrel{d}{=}\left(v\left(x_{1}\right), \ldots, v\left(x_{n}\right)\right)
$$

as random vectors for every finite collection of points $x_{1}, \ldots, x_{n} \in \mathbb{R}^{d}$.

Definition 2.1. Let $1 \leq p \leq \infty$. Consider the group $\Phi$ of isometries on $\mathbb{R}^{d}$ and a random field $u \in C_{b}\left(\mathbb{R}^{d}, L^{p}(\Omega)\right)$. For a given function $\varphi \in \Phi$ let $(\varphi u)(x)=$ $u(\varphi(x))$. We define the space Iso $_{p}$ of isometry-invariant random fields with finite $p$-th moment as

$$
\operatorname{Iso}_{p}=\left\{u \in C_{b}\left(\mathbb{R}^{d}, L^{p}(\Omega)\right): u \stackrel{d}{=} \varphi u \text { for every } \varphi \in \Phi\right\} .
$$

Remark 2.2. Notice that the space Iso $_{p}$ inherits the norm (and hence topology) of the space $C_{b}\left(\mathbb{R}^{d}, L^{p}(\Omega)\right)$ and it forms a closed subspace therein. Indeed, fix an isometry $\varphi \in \Phi$ and a sequence $u_{n} \in \mathrm{Iso}_{p}$ such that $\lim _{n \rightarrow \infty}\left\|u_{n}-u\right\|_{p}=0$. Then $u_{n} \stackrel{d}{=} \varphi u_{n}$ and because

$$
\left\|\varphi u_{n}-\varphi u\right\|_{p}=\sup _{x \in \mathbb{R}^{d}}\left\|u_{n}(\varphi(x))-u(\varphi(x))\right\|_{L^{p}(\Omega)}=\left\|u_{n}-u\right\|_{p}
$$

we have $u \stackrel{d}{=} \varphi u$, hence $u \in \operatorname{Iso}_{p}$. This also entails completeness.

Remark 2.3. Notice that the property $u \stackrel{d}{=} \varphi u$ implies that neither $\|u(x)\|_{p}$ nor $E u(x)$ depend on the $x$ variable.

Remark 2.4. The space Iso $_{p}$ is not linear. For a simple example, let $X, Y$ be two independent random variables with identical distribution $\mathcal{N}(0,1)$. Define random fields $u, v \in C_{b}\left(\mathbb{R}, L^{2}(\Omega)\right)$ as $u(x)=X$ and $v(x)=\sin (x) X+\cos (x) Y$. Notice that $u, v \in \mathrm{Iso}_{2}$. However, by calculating

$$
E(u(x)+v(x))^{2}=2 \sin (x)+2
$$

we discover that $u+v \notin \mathrm{Iso}_{2}$ (cf. Remark 2.3).

In the particular case of $p=2$, for every random field $u \in \mathrm{IsO}_{2}$ we have $E u(x) u(y)=B(|x-y|)$ for a certain positive definite function $B \in C(\mathbb{R}, \mathbb{R})$. Moreover we have the following representation

$$
u(x)=E u(x)+\int_{\mathbb{R}^{d}} e^{i x \cdot \xi} Z(d \xi),
$$

where $Z$ is an orthogonal random measure (see [IL89, Chapter 1] for specific results as well as GV64, GS69, or Rao12 for general theory of random measures). There also exists a finite measure $\sigma$ such that

$$
E(u(x))^{2}-(E u(x))^{2}=E\left(\int_{\mathbb{R}^{d}} e^{i x \cdot \xi} Z(d \xi)\right)^{2}=\int_{\mathbb{R}^{d}} \sigma(d \xi)=\sigma\left(\mathbb{R}^{d}\right) .
$$

In this case we denote $\sigma=\operatorname{Spec}(u)$. One may also observe that $\mathcal{F}(\sigma)=B$. 
As in the general case (2), for every $K \geq 0$ we define the Bielecki norm and the corresponding space

$$
\|u\|\left\|_{K, p}=\sup _{t \geq 0} e^{-t K}\right\| u(t) \|_{p}, \quad \mathcal{B}_{K, p}=\left\{u \in C\left([0, \infty), \mathrm{Iso}_{p}\right):\|u\| \|_{K, p}<\infty\right\} .
$$

We use these to introduce the spaces of jointly isometry-invariant random fields.

Definition 2.5. Given an isometry $\varphi \in \Phi$ and $u \in C\left([0, \infty)\right.$, Iso $\left._{p}\right)$ let us set $(\varphi u)(t, x)=u(t, \varphi(x))$. For $K \geq 0$ and $p \geq 1$ we define

$$
\mathrm{J}_{-\mathrm{IsO}_{K, p}}=\left\{u \in \mathcal{B}_{K, p}: u \stackrel{d}{=} \varphi u \text { for every } \varphi \in \Phi\right\} .
$$

Similarly to the case of $\mathrm{Iso}_{p}$, the space $\mathrm{J}-\mathrm{Iso}_{K, p}$ is not linear, but it is complete in the norm we defined. In order to compensate for the lack of linearity we consider the following relation.

Definition 2.6. Let $u, v \in \mathrm{J}^{-\mathrm{Iso}_{K, p}}$ or $u, v \in \mathrm{Iso}_{p}$. We say that $u \sim v$ if for every isometry $\varphi \in \Phi$ we have $(u, v) \stackrel{d}{=}(\varphi u, \varphi v)$.

It immediately implies that when $u, v \in \mathrm{J}^{-\mathrm{Iso}_{K, p}}$ such that $u \sim v$, we have $\alpha u+\beta v \in \mathrm{J}^{-\mathrm{Iso}_{K, p}}$ for every $\alpha, \beta \in \mathbb{R}$. The same observation holds in the space Iso $_{p}$. Keep in mind that this relation is not transitive.

Remark 2.7. Let $u, v \in \mathrm{J}^{-\mathrm{Iso}_{K, p}}$ or $u, v \in \mathrm{Iso}_{p}$ and $u \sim v$, and take two measurable functions $f$ and $g$. Notice that because $(u, v) \stackrel{d}{=}(\varphi u, \varphi v)$, we also have (see Bil95. Theorem 25.7])

$$
(f(u, v), g(u, v)) \stackrel{d}{=}(f(\varphi u, \varphi v), g(\varphi u, \varphi v))
$$

and therefore $f(u, v) \sim g(u, v)$. By similar arguments we have $f(u) \sim f(v)$ and $f(u) \sim g(u)$.

Remark 2.8. Let $u \in \mathrm{J}^{-\mathrm{Iso}_{K, p}} \cap C^{1}\left([0, \infty), C_{b}\left(\mathbb{R}^{d}, L^{p}(\Omega)\right)\right)$ and $t, h \geq 0$. Notice that $u(t) \sim u(t+h)$, hence $u(t+h)-u(t) \in \mathrm{Iso}_{p}$. We may consider

$$
\partial_{t} u(t)=\lim _{h \rightarrow 0} \frac{u(t+h)-u(t)}{h}
$$

where the limit is taken in the sense of the norm $\|\cdot\|_{p}$, and we have $\varphi\left(\partial_{t} u\right) \stackrel{d}{=} \partial_{t} u$ for every isometry $\varphi \in \Phi$.

Definition 2.9. Let $u \in C_{b}\left(\mathbb{R}^{d}, L^{p}(\Omega)\right)$. For a given $z \in \mathbb{R}^{d}$ we define the (normalised) directional derivative $\nabla_{z} u$ as the random field such that

$$
\lim _{h \rightarrow 0}\left\|\frac{u(x+h z)-u(x)}{h|z|}-\nabla_{z} u(x)\right\|_{p}=0,
$$

whenever the limit exists. We say that $u \in C_{b}^{1}\left(\mathbb{R}^{d}, L^{p}(\Omega)\right)$ if for every $z \in \mathbb{R}^{d}$ we have $\nabla_{z} u \in C_{b}\left(\mathbb{R}^{d}, L^{p}(\Omega)\right)$.

Remark 2.10. Suppose that $u \in \mathrm{IsO}_{2}$ has the repesentation $u(x)=E u(x)+$ $\int_{\mathbb{R}^{d}} e^{i x \cdot \xi} Z(d \xi)$ and $\nabla_{z} u$ exists. It follows directly from identity (5) that we have

$$
\nabla_{z} u(x)=\int_{\mathbb{R}^{d}} i \frac{z}{|z|} \cdot \xi e^{i x \cdot \xi} Z(d \xi) .
$$

Lemma 2.11. Let $u, v \in \mathrm{IsO}_{2}$ and $u \sim v$. If $u \in C_{b}^{1}\left(\mathbb{R}^{d}, L^{2}(\Omega)\right)$ then for every $z \in \mathbb{R}^{d}$ we have

$$
E \nabla_{z} u(x) v(x)=0 .
$$


Proof. Since we assume $u \sim v$, hence for every $x, y \in \mathbb{R}^{d}$ we have $E u(y) v(0)=$ $E u(-y) v(0)$ and $E u(x+y) v(x)=E u(y) v(0)$. Therefore

$$
\begin{array}{r}
E \nabla_{z} u(x) v(x)=\lim _{h \rightarrow 0} E \frac{u(x+h z)-u(x)}{h|z|} v(x)=\lim _{h \rightarrow 0} E \frac{u(h z)-u(0)}{h|z|} v(0) \\
=\lim _{h \rightarrow 0} E \frac{u(-h z)-u(0)}{h|z|} v(0)=E \nabla_{-z} u(x) v(x) .
\end{array}
$$

On the other hand we have $\nabla_{z} u=-\nabla_{-z} u$, hence $E \nabla_{z} u(x) v(x)=0$.

2.3. Spectral moments. We introduce Sobolev-type spaces of isotropic random fields.

Definition 2.12. For every $\alpha \geq 0$ we define the space

$$
\mathrm{Iso}_{2}^{\alpha}=\left\{u_{0} \in \mathrm{IsO}_{2}: \int_{\mathbb{R}^{d}}|\xi|^{2 \alpha} d \sigma(\xi)<\infty, \text { where } \sigma=\operatorname{Spec}\left(u_{0}\right)\right\}
$$

supplemented with the norm $\left\|u_{0}\right\|_{\alpha, 2}^{2}=\int_{\mathbb{R}^{d}}\left(1+|\xi|^{2 \alpha}\right) d \sigma(\xi)$.

Proposition 2.13. We have the following embeddings

(1) $\operatorname{Iso}_{2}^{1} \subset C_{b}^{1}\left(\mathbb{R}^{d}, L^{2}(\Omega)\right)$

(2) $\mathrm{Iso}_{2}^{\alpha} \subset \mathrm{Iso}_{2}^{\beta}$ for every $\alpha \geq \beta$.

Proof. Let $\sigma$ be the spectral measure and $Z$ be the orthogonal random measure corresponding to an arbitrary $u_{0} \in \mathrm{IsO}_{2}$.

Suppose $u_{0} \in \mathrm{Iso}_{2}^{1}$ and take an arbitrary $z \in \mathbb{R}^{d}$. Then it follows from the Cauchy-Schwarz inequality and Remark 2.10 that

$$
\begin{aligned}
E\left(\nabla_{z} u_{0}(x)\right)^{2}=E\left(\int_{\mathbb{R}^{d}} i \frac{z}{|z|} \cdot \xi e^{i x \cdot \xi} Z(d \xi)\right)^{2} & \\
& =\int_{\mathbb{R}^{d}}\left|i \frac{z}{|z|} \cdot \xi\right|^{2} d \sigma(\xi) \leq \int_{\mathbb{R}^{d}}|\xi|^{2} d \sigma(\xi) \leq\left\|u_{0}\right\|_{1,2}^{2} .
\end{aligned}
$$

The continuity follows from a similar calculation and the Lebesgue dominated convergence theorem. This concludes the proof of the first inclusion.

To prove the second claim it suffices to notice that the measure $\sigma$ is finite and for $\alpha \geq \beta$ the function $\psi(|x|)=|x|^{\beta / \alpha}$ is concave. Therefore by the Jensen inequality we have

$$
\frac{1}{\sigma\left(\mathbb{R}^{d}\right)} \int_{\mathbb{R}^{d}} \psi\left(|\xi|^{2 \alpha}\right) d \sigma(\xi) \leq \psi\left(\frac{1}{\sigma\left(\mathbb{R}^{d}\right)} \int_{\mathbb{R}^{d}}|\xi|^{2 \alpha} d \sigma(\xi)\right),
$$

hence $\left\|u_{0}\right\|_{\beta, 2} \leq C\left\|u_{0}\right\|_{\alpha, 2}$ for some constant $C>0$.

Lemma 2.14. Let $u \in \mathrm{IsO}_{2}$. We have $u \in \mathrm{Iso}_{2}^{1}$ if and only if there exist $c>0$ and $\epsilon>0$ such that

$$
E\left|\frac{u(x+h z)-u(x)}{h|z|}\right|^{2} \leq c
$$

for every $h \in(0, \epsilon)$ and every $z \in \mathbb{R}^{d}$.

Proof. Let $\sigma=\operatorname{Spec}(u)$. Since we assume $u \in \mathrm{IsO}_{2}$, we have

$$
\begin{aligned}
& E\left|\frac{u(x+h z)-u(x)}{h|z|}\right|^{2}=E\left|\int_{\mathbb{R}^{d}} \frac{e^{i(x+h z) \cdot \xi}-e^{i x \cdot \xi}}{h|z|} Z(d \xi)\right|^{2} \\
&=\int_{\mathbb{R}^{d}}\left|\frac{e^{i(x+h z) \cdot \xi}-e^{i x \cdot \xi}}{h|z|}\right|^{2} d \sigma(\xi) .
\end{aligned}
$$


Suppose that (6) holds for some $c>0$, every $h \in(0, \epsilon)$ and every $z \in \mathbb{R}^{d}$. The Fatou lemma gives us

$$
\int_{\mathbb{R}^{d}}|\xi|^{2} d \sigma(\xi) \leq \liminf _{h \rightarrow 0} \int_{\mathbb{R}^{d}}\left|\frac{e^{i(x+h z) \cdot \xi}-e^{i x \cdot \xi}}{h|z|}\right|^{2} d \sigma(\xi),
$$

which implies

$$
\int_{\mathbb{R}^{d}}|\xi|^{2} d \sigma(\xi) \leq c,
$$

hence $u \in \mathrm{Iso}_{2}^{1}$. Now suppose that $u \in \mathrm{Iso}_{2}^{1}$. By definition we have $\int_{\mathbb{R}^{d}}|\xi|^{2} d \sigma(\xi)=$ $\|u\|_{1,2}^{2}-\|u\|_{2}^{2}$. Because of a simple inequality $\left|e^{i x \cdot y}-1\right| \leq|x \cdot y| \leq|x||y|$ for every $x, y \in \mathbb{R}^{d}$, we have

$$
\left|\frac{e^{i(x+h z) \cdot \xi}-e^{i x \cdot \xi}}{h|z|}\right|^{2} \leq|\xi|^{2}
$$

and therefore

$$
\int_{\mathbb{R}^{d}}\left|\frac{e^{i(x+h z) \cdot \xi}-e^{i x \cdot \xi}}{h|z|}\right|^{2} d \sigma(\xi) \leq\|u\|_{1,2}^{2}-\|u\|_{2}^{2} .
$$

Combining this with (7) we get the estimate (6).

Corollary 2.15. If $u \in \mathrm{IsO}_{2}^{1}$ and $f: \mathbb{R} \rightarrow \mathbb{R}$ is Lipschitz then $f(u) \in \mathrm{Iso}_{2}^{1}$.

Proof. Because $f$ is Lipschitz we have

$$
\left\|\frac{f(u(x+h z))-f(u(x))}{h|z|}\right\|_{2} \leq L\left\|\frac{u(x+h z)-u(x)}{h|z|}\right\|_{2} .
$$

We conclude by applying Lemma 2.14 twice.

\section{Linear EQUATion}

In this section we discuss properties of solutions to the linear Cauchy problem

$$
\left\{\begin{array}{lr}
\partial_{t} u+(-\Delta)^{s} u=0 & \text { on }[0, \infty) \times \mathbb{R}^{d}, \\
u(0) \stackrel{d}{=} u_{0} & \text { on } \mathbb{R}^{d} .
\end{array}\right.
$$

Before we study the solutions themselves we need to describe in detail some of the objects we work with.

Definition 3.1. Let $u \in C_{c}^{\infty}\left(\mathbb{R}^{d}, \mathbb{R}\right)$ and $s \in(0,1]$. We define the fractional Laplace operator

$$
(-\Delta)^{s} u(x)=\mathcal{F}\left\{|\xi|^{2 s} \mathcal{F}^{-1} u(\xi)\right\} .
$$

Definition 3.2. For $u_{0} \in \mathrm{Iso}_{2}^{2 s}$, such that $u_{0}=E u_{0}(x)+\int_{\mathbb{R}^{d}} e^{i x \cdot \xi} Z(d \xi)$, we define

$$
-(-\Delta)^{s} u_{0}(x)=\int_{\mathbb{R}^{d}}|\xi|^{2 s} e^{i x \cdot \xi} Z(d \xi) .
$$

Remark 3.3. Notice that we have

$$
E(-\Delta)^{s} u_{0}(x)=0
$$

and $\operatorname{Spec}\left((-\Delta)^{s} u_{0}\right)=|\xi|^{4 s} \sigma(d \xi)$.

By $\left\{P_{t}\right\}_{t \geq 0}$ we denote the usual semigroup of linear operators generated by the fractional Laplacian (regardless of the chosen parameter $s$, which we assume to be fixed). We define the action of the operators $P_{t}$ on the space of isometry-invariant random fields in the following fashion. 
Definition 3.4. For $u_{0} \in \mathrm{Iso}_{2}$, such that $u_{0}(x)=E u_{0}(x)+\int_{\mathbb{R}^{d}} e^{i x \cdot \xi} Z(d \xi)$, we define

$$
P_{t} u_{0}(x)=E u_{0}(x)+\int_{\mathbb{R}^{d}} e^{i x \cdot \xi-t|\xi|^{2 s}} Z(d \xi) .
$$

Remark 3.5. Notice that

$$
\left\|P_{t} u_{0}\right\|_{2}^{2}=\left(E u_{0}(x)\right)^{2}+\int_{\mathbb{R}^{d}} e^{-2 t|\xi|^{2 s}} \sigma(d \xi) \leq\left\|u_{0}\right\|_{2}^{2},
$$

which shows that $P_{t}$ is a contractive operator on $\mathrm{Iso}_{2}$. This expression also shows that $\operatorname{Spec}\left(P_{t} u_{0}\right)=e^{-2 t|\xi|^{2 s}} \sigma(d \xi)$. By comparing Definition 3.4 with representation (3i) we see that the semigroup property is preserved as well

$$
\begin{aligned}
P_{r}\left(P_{t} u_{0}(x)\right)=E P_{t} u_{0}(x)+\int_{\mathbb{R}^{d}} e^{i x \cdot \xi-r|\xi|^{2 s}}\left(e^{-t|\xi|^{2 s}} Z(d \xi)\right) \\
=E u_{0}(x)+\int_{\mathbb{R}^{d}} e^{i x \cdot \xi-(r+t)|\xi|^{2 s}} Z(d \xi)=P_{r+t} u_{0}(x) .
\end{aligned}
$$

Proposition 3.6. If $u_{0} \in \mathrm{IsO}_{2}^{s}$ then

$$
\lim _{h \rightarrow 0} E \frac{u_{0}-P_{h} u_{0}}{h} u_{0}=E\left((-\Delta)^{s / 2} u_{0}\right)^{2} .
$$

Proof. Let $\sigma$ be the spectral measure and $Z$ be the orthogonal random measure corresponding to $u_{0}$. We have

$$
\begin{aligned}
E \frac{u_{0}-P_{h} u_{0}}{h} u_{0}=\frac{1}{h} E \int_{\mathbb{R}^{d}}\left(e^{i x \cdot \xi}-e^{i x \cdot \xi-h|\xi|^{2 s}}\right) & Z(d \xi) \int_{\mathbb{R}^{d}} e^{i x \cdot \xi} Z(d \xi) \\
& =\frac{1}{h} \int_{\mathbb{R}^{d}}\left(1-e^{-h|\xi|^{2 s}}\right) \sigma(d \xi) .
\end{aligned}
$$

Because we assume $u_{0} \in \mathrm{Iso}_{2}^{s}$ we may use the Lebesgue dominated convergence theorem and pass to the limit to obtain

$$
\lim _{h \rightarrow 0} \frac{1}{h} \int_{\mathbb{R}^{d}}\left(1-e^{-h|\xi|^{2 s}}\right) \sigma(d \xi)=\int_{\mathbb{R}^{d}}|\xi|^{2 s} \sigma(d \xi) .
$$

On the other hand,

$$
\int_{\mathbb{R}^{d}}|\xi|^{2 s} \sigma(d \xi)=E\left(\int_{\mathbb{R}^{d}}|\xi|^{s} e^{i x \cdot \xi} Z(d \xi)\right)^{2}=E\left((-\Delta)^{s / 2} u_{0}\right)^{2}
$$

(cf. Remark 3.3).

In addition to such "spectral" framework we may employ a direct approach as well. To this end we introduce the kernel $p_{t}$ of the operator $P_{t}$ defined by the formula

$$
p_{t}(x)=\int_{\mathbb{R}^{d}} e^{i x \cdot \xi-t|\xi|^{2 s}} d \xi .
$$

It is well-known that for $s \in(0,1]$ and $t \geq 0$ the function $p_{t}$ is positive, radially symmetric and the function $t \mapsto p_{t}(y)$ is continuous. For every $t>0$ we also have $\int_{\mathbb{R}^{d}} p_{t}(x) d x=1$.

The following lemma provides a connection between the two approaches.

Lemma 3.7. Let $u_{0} \in \mathrm{Iso}_{p}$ for some $2 \leq p \leq \infty$. Then for every $t>0$ we have

$$
P_{t} u_{0}(x)=\int_{\mathbb{R}^{d}} p_{t}(y) u_{0}(x-y) d y,
$$

where the integral is understood in the Bochner sense on functions in the space $C_{b}\left(\mathbb{R}^{d}, L^{p}(\Omega)\right)$. Moreover, $\left\|P_{t} u_{0}\right\|_{p} \leq\left\|u_{0}\right\|_{p}$ for all $t \geq 0$. 
Proof. Let $u_{0}(x)=E u_{0}(x)+\int_{\mathbb{R}^{d}} e^{i x \cdot \xi} Z(d \xi)$. Following the general definition we have

$$
\begin{aligned}
P_{t} u_{0}(x)=E u_{0}(x)+\int_{\mathbb{R}^{d}} e^{i x \cdot \xi-t|\xi|^{2 s}} Z(d \xi) & =E u_{0}(x)+\int_{\mathbb{R}^{d}} \int_{\mathbb{R}^{d}} p_{t}(y) e^{-i y \xi} d y e^{i x \cdot \xi} Z(d \xi) .
\end{aligned}
$$

By the Fubini theorem and the fact that $\int_{\mathbb{R}^{d}} p_{t}(y) d y=1$ we then obtain

$$
\begin{aligned}
& P_{t} u_{0}(x)=E u_{0}(x)+\int_{\mathbb{R}^{d}} p_{t}(y) \int_{\mathbb{R}^{d}} e^{-i y \xi} e^{i x \cdot \xi} Z(d \xi) d y \\
& \quad=\int_{\mathbb{R}^{d}} p_{t}(y)\left(E u_{0}(x)+\int_{\mathbb{R}^{d}} e^{i(x-y) \cdot \xi} Z(d \xi)\right) d y=\int_{\mathbb{R}^{d}} p_{t}(y) u_{0}(x-y) d y .
\end{aligned}
$$

The last integral is convergent in the Bochner sense because we have

$$
\int_{\mathbb{R}^{d}}\left\|p_{t}(y) u_{0}(x-y)\right\|_{L^{p}(\Omega)} d y=\left\|u_{0}\right\|_{p} \int_{\mathbb{R}^{d}} p_{t}(y) d y=\left\|u_{0}\right\|_{p},
$$

which also confirms that $\left\|P_{t} u_{0}\right\|_{p} \leq\left\|u_{0}\right\|_{p}$.

Proposition 3.8. If $u_{0} \in \mathrm{Iso}_{\infty}$ then $P_{t} u_{0}(x, \omega)=\int_{\mathbb{R}^{d}} p_{t}(y) u_{0}(x-y, \omega) d y$ for every $t>0$ and almost every $\omega \in \Omega$.

Proof. Notice that the operator $T_{\omega}: C\left(\mathbb{R}^{d}, L^{\infty}(\Omega)\right) \rightarrow L^{\infty}\left(\mathbb{R}^{d}, \operatorname{Bor}\left(\mathbb{R}^{d}\right), d x\right)$ defined as $T_{\omega} u=u(\cdot, \omega)$ is bounded. By the Hille theorem we then have

$$
\begin{aligned}
P_{t} u_{0}(x, \omega)=T_{\omega} & \left(\int_{\mathbb{R}^{d}} p_{t}(y) u_{0}(x-y) d y\right) \\
& =\int_{\mathbb{R}^{d}} p_{t}(y) T_{\omega}\left(u_{0}(x-y)\right) d y=\int_{\mathbb{R}^{d}} p_{t}(y) u_{0}(x-y, \omega) d y .
\end{aligned}
$$

Remark 3.9. Notice that without additional assumptions, an individual realisation $u_{0}(x, \omega)$ of the random field $u_{0}$ may not be integrable, such that for a given $\omega \in \Omega$, the Lebesgue integral $\int_{\mathbb{R}^{d}} p_{t}(y) u_{0}(x-y, \omega) d y$ may not exist. This is the main reason why we cannot consider solutions for every single realisation of the initial data separately and then average them out to get our results.

Lemma 3.10. For every $2 \leq p \leq \infty$ and every $K \geq 0$ if $u_{0} \in \operatorname{Iso}_{p}$ then $P_{t} u_{0} \in$ $\mathrm{J}^{-\mathrm{ISO}_{K, p}}$.

Proof. By Lemma 3.7 we have

$$
P_{t} u_{0}(x)=\int_{\mathbb{R}^{d}} p_{t}(y) u_{0}(x-y) d y
$$

Consider a sequence $t_{n} \rightarrow t$. By the continuity of the function $t \mapsto p_{t}(y)$ and the Lebesgue dominated convergence theorem we obtain

$$
\begin{aligned}
\lim _{n \rightarrow \infty}\left\|P_{t} u_{0}(x)-P_{t_{n}} u_{0}(x)\right\|_{p} \leq & \lim _{n \rightarrow \infty} \int_{\mathbb{R}^{d}}\left\|\left(p_{t}(y)-p_{t_{n}}(y)\right) u_{0}(x-y)\right\|_{p} \\
& =\left\|u_{0}\right\|_{p} \lim _{n \rightarrow \infty} \int_{\mathbb{R}^{d}}\left|\left(p_{t}(y)-p_{t_{n}}(y)\right)\right|=0 .
\end{aligned}
$$

For every $K \geq 0$ and $2 \leq p \leq \infty$ identity (9) gives us the estimate

$$
\sup _{t \geq 0} e^{-t K}\left\|P_{t} u_{0}(x)\right\|_{p} \leq \sup _{t \geq 0} e^{-t K} \int_{\mathbb{R}^{d}}\left\|p_{t}(y) u_{0}(x-y)\right\|_{p} d y \leq\left\|u_{0}\right\|_{p},
$$

which shows that $P_{t} u_{0} \in \mathcal{B}_{K, p}$. 
Let $\varphi \in \Phi$ be an isometry and $Z$ be the orthogonal random measure corresponding to $u_{0}$. Then because $u_{0} \in \mathrm{Iso}_{2}$ we have

$$
\begin{aligned}
\varphi\left(P_{t} u_{0}\right)(x)=\varphi\left(\int_{\mathbb{R}^{d}} e^{i x \cdot \xi-t|\xi|^{2 s}} Z(d \xi)\right) \\
=\int_{\mathbb{R}^{d}} e^{i \varphi(x) \cdot \xi-t|\xi|^{2 s}} Z(d \xi)=P_{t} u_{0}(\varphi(x)) \stackrel{d}{=} P_{t} u_{0}(x),
\end{aligned}
$$

which confirms that $P_{t} u_{0} \in \mathrm{J}_{-\mathrm{Iso}_{K, p}}$ (see Definition 2.5).

Let us now show a regularising effect of the linear semigroup.

Lemma 3.11. If $u_{0} \in \mathrm{Iso}_{2}$ then $P_{t} u_{0} \in C\left((0, \infty), \mathrm{Iso}_{2}^{\alpha}\right)$ for every $\alpha \geq 0$. Moreover there exists a constant $c_{s, \alpha}$ such that for every $t>0$

$$
\left\|P_{t} u_{0}\right\|_{\alpha, 2} \leq\left(1+c_{s, \alpha} t^{-\alpha / 2 s}\right)\left\|u_{0}\right\|_{2} .
$$

Proof. Let $\sigma=\operatorname{Spec}\left(u_{0}\right)$. Keep in mind it is a finite measure and $\sigma\left(\mathbb{R}^{d}\right)=\left\|u_{0}\right\|_{2}^{2}$. We then have $\operatorname{Spec}\left(P_{t} u_{0}\right)=e^{-2 t|\xi|^{2 s}} \sigma(d \xi)$ and

$$
\left\|P_{t} u_{0}\right\|_{\alpha, 2}^{2}=\int_{\mathbb{R}^{d}}\left(1+|\xi|^{2 \alpha}\right) e^{-2 t|\xi|^{2 s}} \sigma(d \xi) \leq\left(1+\sup _{\xi \in \mathbb{R}^{d}}|\xi|^{2 \alpha} e^{-2 t|\xi|^{2 s}}\right) \sigma\left(\mathbb{R}^{d}\right) .
$$

Notice that for every $t>0$ and $\alpha \geq 0$ we have

$$
\sup _{\xi \in \mathbb{R}^{d}}|\xi|^{2 \alpha} e^{-2 t|\xi|^{2 s}}=(2 t)^{-\alpha / s} \sup _{\xi \in \mathbb{R}^{d}}|\xi|^{2 \alpha} e^{-|\xi|^{2 s}}=c_{s, \alpha}^{2} t^{-\alpha / s},
$$

which shows that

$$
\left\|P_{t} u_{0}\right\|_{\alpha, 2} \leq\left\|u_{0}\right\|_{2} \sqrt{1+c_{s, \alpha}^{2} t^{-\alpha / s}} \leq\left\|u_{0}\right\|_{2}\left(1+c_{s, \alpha} t^{-\alpha / 2 s}\right) .
$$

Finally, by the Lebesgue dominated convergence theorem, for every $t>0$ we obtain

$$
\lim _{\tau \rightarrow t}\left\|P_{t} u_{0}-P_{\tau} u_{0}\right\|_{\alpha, 2}^{2}=\lim _{\tau \rightarrow t} \int_{\mathbb{R}^{d}}\left(1+|\xi|^{2 \alpha}\right)\left(e^{-2 t|\xi|^{2 s}}-e^{-2 \tau|\xi|^{2 s}}\right) d \sigma(\xi)=0,
$$

which confirms the continuity.

Lemma 3.12. If $u_{0} \in \mathrm{Iso}_{2}^{\alpha}$ then $\nabla_{z} P_{t} u_{0} \in C\left((0, \infty), \mathrm{Iso}_{2}^{\alpha}\right)$ for every $\alpha \geq 0$. Moreover there exists a constant $c_{s}$ (independent of $\alpha$ ) such that for every $t>0$

$$
\left\|\nabla_{z} P_{t} u_{0}\right\|_{\alpha, 2} \leq c_{s} t^{-1 / 2 s}\left\|u_{0}\right\|_{\alpha, 2} .
$$

Proof. The proof is almost identical to that of Lemma 3.11 Let $\sigma=\operatorname{Spec}\left(u_{0}\right)$. Then, because of the Cauchy-Schwarz inequality and identity (10), we have

$$
\begin{aligned}
\left\|\nabla_{z} P_{t} u_{0}\right\|_{\alpha, 2}^{2}= & \int_{\mathbb{R}^{d}}\left(1+|\xi|^{2 \alpha}\right)\left|\frac{z}{|z|} \cdot \xi\right|^{2} e^{-2 t|\xi|^{2 s}} \sigma(d \xi) \\
& \leq \sup _{\xi \in \mathbb{R}^{d}}|\xi|^{2} e^{-2 t|\xi|^{2 s}} \int_{\mathbb{R}^{d}}\left(1+|\xi|^{2 \alpha}\right) \sigma(d \xi)=c_{s}^{2} t^{-1 / s}\left\|u_{0}\right\|_{\alpha, 2}^{2} .
\end{aligned}
$$

The continuity follows in a similar fashion.

Lemma 3.13. There exists a constant $c_{s}$ (independent of $p$ ) such that if $u_{0} \in \mathrm{Iso}_{p}$ then

$$
\left\|\nabla_{z} P_{t} u_{0}\right\|_{p} \leq c_{s} t^{-1 / 2 s}\left\|u_{0}\right\|_{p}
$$

for every $t>0$. 
Proof. Notice that

$$
\nabla_{z} P_{t} u_{0}=\int_{\mathbb{R}^{d}} \nabla_{z} p_{t}(y) u_{0}(x-y) d y .
$$

It is well-known that (see KW08 or BPSV14])

$$
p_{t}(y)=t^{-d / 2 s} p_{1}\left(t^{-1 / 2 s} y\right)
$$

for every $t>0$ and

$$
\left|\nabla_{z} p_{1}(y)\right| \leq C(1+|y|)^{-(2 s+d+1)} .
$$

This allows us to estimate

$$
\begin{gathered}
\left\|\nabla_{z} P_{t} u_{0}\right\|_{p} \leq \int_{\mathbb{R}^{d}}\left\|\nabla_{z} p_{t}(y) u_{0}(x-y)\right\|_{p} d y=\left\|u_{0}\right\|_{p} \int_{\mathbb{R}^{d}}\left|\nabla_{z} p_{t}(y)\right| d y \\
\leq t^{-(d+1) / 2 s}\left\|u_{0}\right\|_{p} C \int_{\mathbb{R}^{d}}\left(1+\left|t^{-1 / 2 s} y\right|\right)^{-(2 s+d+1)} d y \\
=t^{-1 / 2 s}\left\|u_{0}\right\|_{p} C \int_{\mathbb{R}^{d}}(1+|y|)^{-(2 s+d+1)} d y .
\end{gathered}
$$

The last integral is convergent and does not depend on $p$.

The following theorem justifies calling $P_{t} u_{0}$ a solution to problem (8).

Theorem 3.14. If $u_{0} \in \mathrm{Iso}_{2}$ and $u(t)=P_{t} u_{0}$ then $u \in C^{1}\left((0, \infty), \mathrm{Iso}_{2}\right), \partial_{t} u \in$ $\mathrm{J}_{-\mathrm{Iso}_{K, 2}}$ for every $K \geq 0$ and

$$
\partial_{t} u+(-\Delta)^{s} u=0 \text { for every } t>0 .
$$

Proof. It follows from Lemma 3.11 that $(-\Delta)^{s} u(t) \in \mathrm{Iso}_{2}$ for every $t>0$. Let $\sigma=\operatorname{Spec}\left(u_{0}\right)$. Then $\operatorname{Spec}(u(t))=e^{-2 t|\xi|^{2 s}} \sigma(d \xi)$ and due to identity (4) and Definition 3.2 it suffices to consider

$$
E\left|\frac{u(t+h)-u(t)}{h}+(-\Delta)^{s} u(t)\right|^{2}=\int_{\mathbb{R}^{d}}\left(\frac{e^{-h|\xi|^{2 s}}-1}{h}-|\xi|^{2 s}\right)^{2} e^{-2 t|\xi|^{2 s}} d \sigma(\xi) .
$$

Since $\sigma$ is a finite measure we may pass to the limit with $h \rightarrow 0$ on both sides and use the Lebesgue dominated convergence theorem to obtain

$$
\partial_{t} u(t)=-(-\Delta)^{s} u(t)
$$

for every $t>0$. Moreover, we have (see estimate (10))

$$
\left\|\partial_{t} u(t)\right\|_{2}^{2}=\int_{\mathbb{R}^{d}}|\xi|^{4 s} e^{-2 t|\xi|^{2 s}} \sigma(d \xi) \leq c_{s} t^{-2}\left\|u_{0}\right\|_{2}^{2},
$$

therefore $\partial_{t} u \in \mathrm{J}^{-\mathrm{IsO}_{K, 2}}$ for every $K \geq 0$.

Remark 3.15. The analysis of the linear problem is exposed in more detail in Kru17. However, here we use a different definition of solutions, which is better suited for the nonlinear case which we discuss in the sequel.

\section{Equation With Lipschitz NONLINEARITY}

Let us consider the following initial value problem

$$
\left\{\begin{array}{lr}
\partial_{t} u+(-\Delta)^{s} u=\nabla_{z} f(u) & \text { on }[0, \infty) \times \mathbb{R}^{d}, \\
u(0) \stackrel{d}{=} u_{0} & \text { on } \mathbb{R}^{d} .
\end{array}\right.
$$

Here we assume $s \in\left(\frac{1}{2}, 1\right], u_{0} \in$ Iso $_{p}$ and the function $f: \mathbb{R} \rightarrow \mathbb{R}$ is to be Lipschitz, i.e. for every $x, y \in \mathbb{R}$ and some constant $L>0$ we have $|f(x)-f(y)| \leq L|x-y|$, and such that $f(0)=0$. In the following, by referring to problem (11), we also quietly include these assumptions. 


\subsection{Existence of solutions.}

Definition 4.1. Given $u \in \mathrm{J}^{-\mathrm{Iso}_{K, 2}}$ for some $K \geq 0$, we define the following nonlinear operator

$$
F(u)(t)=P_{t} u(0)+\int_{0}^{t} \nabla_{z} P_{t-\tau} f(u(\tau)) d \tau .
$$

Let $u_{0} \in \mathrm{Iso}_{p}$. For $K \geq 0$ we say that $u \in \mathrm{J}^{-\mathrm{Iso}_{K, p}}$ is a solution to problem (11) if $F(u)=u$ and $u_{0} \stackrel{d}{=} u(0)$.

Lemma 4.2. For every $K>0$ and $2 \leq p \leq \infty$ if $u \in \mathrm{J}_{-\mathrm{Iso}_{K, p}}$ then $F(u) \in \mathrm{J}^{-\mathrm{Iso}_{K, p}}$.

Proof. First we estimate the norm to check if $F(u) \in \mathcal{B}_{K, p}$. We have

$$
\|F(u)\|_{K, p}=\sup _{t \geq 0} e^{-t K}\left\|P_{t} u(0)+\int_{0}^{t} \nabla_{z} P_{t-\tau} f(u(\tau)) d \tau\right\|_{p} .
$$

It follows from Lemma 3.13 that

$$
\begin{aligned}
\left\|\int_{0}^{t} \nabla_{z} P_{t-\tau} f(u(\tau)) d \tau\right\|_{p} & \leq c_{s} L \int_{0}^{t}(t-\tau)^{-1 / 2 s}\|u(\tau)\|_{p} d \tau \\
& \leq c_{s} L\left(\sup _{0<\tau<t} e^{-\tau K}\|u(\tau)\|_{p}\right) \int_{0}^{t}(t-\tau)^{-1 / 2 s} e^{\tau K} d \tau .
\end{aligned}
$$

Using the $\Gamma$ function we estimate

$$
\begin{aligned}
\int_{0}^{t}(t-\tau)^{-1 / 2 s} e^{-K(t-\tau)} d \tau<K^{-1+\frac{1}{2 s}} \int_{0}^{\infty} z^{-1 / 2 s} e^{-z} d z & \\
& =K^{-1+\frac{1}{2 s}} \Gamma\left(1-\frac{1}{2 s}\right),
\end{aligned}
$$

which gives us

$$
\|F(u)\|_{K, p} \leq\left\|P_{t} u_{0}\right\|_{K, p}+c_{s} L K^{-1+\frac{1}{2 s}} \Gamma\left(1-\frac{1}{2 s}\right)\|u\|_{K, p} .
$$

Then for every isometry $\varphi \in \Phi$ we observe

$$
\begin{aligned}
& \varphi F(u)=\varphi\left(P_{t} u(0)+\int_{0}^{t} \nabla_{z} P_{t-\tau} f(u(\tau)) d \tau\right) \\
& =\varphi\left(P_{t} u(0)\right)+\varphi\left(\int_{0}^{t} \nabla_{z} P_{t-\tau} f(u(\tau)) d \tau\right) \\
& \quad=P_{t}(\varphi u(0))+\int_{0}^{t} \nabla_{z} P_{t-\tau} f(\varphi u(\tau)) d \tau=F(\varphi u) .
\end{aligned}
$$

Finally, since $\varphi u \stackrel{d}{=} u$, we have $F(\varphi(u)) \stackrel{d}{=} F(u)$ and therefore $\varphi F(u) \stackrel{d}{=} F(u)$.

Lemma 4.3. If $u, v \in \mathrm{J}_{-\mathrm{Iso}_{K, p}}$ and $u \sim v$ then

$$
\|F(u)-F(v)\|_{K, p} \leq\|u(0)-v(0)\|_{p}+c_{s} L K^{-1+\frac{1}{2 s}} \Gamma\left(1-\frac{1}{2 s}\right)\|u-v\|_{K, p} .
$$

Proof. First we notice that $f(u) \sim f(v)$ and therefore

$$
\nabla_{z} P_{t} f(u)-\nabla_{z} P_{t} f(v)=\nabla_{z} P_{t}(f(u)-f(v)) .
$$

This gives us the following inequality

$$
\begin{aligned}
\| F(u)(t)- & F(v)(t) \|_{p} \\
& \leq\left\|P_{t}(u(0)-v(0))\right\|_{p}+\int_{0}^{t}\left\|\nabla_{z} P_{t-\tau}(f(u(\tau))-f(v(\tau)))\right\|_{p} d \tau .
\end{aligned}
$$


Similarly to estimate (12) we have

$$
\begin{aligned}
& \int_{0}^{t} \| \nabla_{z} P_{t-\tau}(f(u(\tau))-f(v(\tau))) \|_{p} d \tau \\
& \leq c_{s} L\left(\sup _{0 \leq \tau \leq t} e^{-\tau K}\|u(\tau)-v(\tau)\|_{p}\right) \int_{0}^{t}(t-\tau)^{-1 / 2 s} e^{\tau K} d \tau .
\end{aligned}
$$

We combine it with estimate (13) and Lemma 3.7 to obtain

$$
\begin{aligned}
& \|F(u)-F(v)\|_{K, p}=\sup _{t \geq 0} e^{-t K}\|F(u)(t)-F(v)(t)\|_{p} \\
& \quad \leq\|u(0)-v(0)\|_{p}+c_{s} L K^{-1+\frac{1}{2 s}} \Gamma\left(1-\frac{1}{2 s}\right)\|u-v\|_{K, p} .
\end{aligned}
$$

Theorem 4.4. Let $\frac{1}{2}<s \leq 1$ and $2 \leq p \leq \infty$. There exists a constant $K_{0}$ such that for every $u_{0} \in \mathrm{Iso}_{p}$ and every $K \geq K_{0}$ the sequence

$$
\left\{\begin{array}{l}
u_{1}=P_{t} u_{0}, \\
u_{n+1}=F\left(u_{n}\right)=F^{n}\left(u_{1}\right)
\end{array}\right.
$$

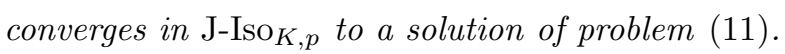

Proof. It follows from Lemma 3.10 that $u_{1} \in \mathrm{J}^{-\mathrm{Iso}_{K, p}}$ for every $K>0$, and then from Lemma 4.2 that $\left\{u_{n}\right\} \subset \mathrm{J}^{-\mathrm{Iso}_{K, p}}$. Let us notice that $u_{n} \sim u_{m}$ and $u_{n}(0)=$ $u_{m}(0)=u_{0}$. Thus by Lemma 4.3 we have

$$
\left\|F\left(u_{n}\right)-F\left(u_{m}\right)\right\|_{K, p} \leq c_{s} L K^{-1+\frac{1}{2 s}} \Gamma\left(1-\frac{1}{2 s}\right)\|\| u_{n}-u_{m} \|_{K, p} .
$$

We now choose such $K_{0}$ that $c_{s} L K_{0}^{-1+\frac{1}{2 s}} \Gamma\left(1-\frac{1}{2 s}\right)<1$, depending on $s$ and $L$. It follows from the Banach fixed point theorem that $\left\{u_{n}\right\}$ is a Cauchy sequence in the space $\mathrm{J}_{-\mathrm{Iso}_{K, p}}$ for every $K \geq K_{0}$ (we use the assumption $s>\frac{1}{2}$ ) and converges to some $u \in \mathrm{J}^{-\mathrm{IsO}_{K, p}}$ which is a fixed point of $F$.

Definition 4.5. We refer to the solution constructed in Theorem 4.4 as the Picard solution.

Corollary 4.6. If $u$ is the Picard solution to (11) then for every $h>0$ and every $t>0$ we have

$$
u(t+h)=P_{h} u(t)+\int_{t}^{t+h} \nabla_{z} P_{t+h-\tau} f(u(\tau)) d \tau .
$$

Proof. Let $u_{n}$ be the sequence of Picard iterations as defined in (14). Since $u_{n}=$ $F\left(u_{n-1}\right)$ for $n \geq 2$ we have

$$
\begin{aligned}
P_{h} u_{n}(t) & =P_{h} P_{t} u_{0}+P_{h} \int_{0}^{t} \nabla_{z} P_{t-\tau} f\left(u_{n-1}(\tau)\right) d \tau \\
& =P_{t+h} u_{0}+\int_{0}^{t} \nabla_{z} P_{t+h-\tau} f\left(u_{n-1}(\tau)\right) d \tau
\end{aligned}
$$

and

$$
u_{n}(t+h)=P_{t+h} u_{0}+\int_{0}^{t+h} \nabla_{z} P_{t+h-\tau} f\left(u_{n-1}(\tau)\right) d \tau .
$$

Hence

$$
\text { opeu }_{n}(t+h)-P_{h} u_{n}(t)=\int_{t}^{t+h} \nabla_{z} P_{t+h-\tau} f\left(u_{n-1}(\tau)\right) d \tau
$$

and finally, because $\lim _{n \rightarrow \infty}\left\|u_{n}-u\right\|_{2}=0$, we get

$$
u(t+h)-P_{h} u(t)=\int_{t}^{t+h} \nabla_{z} P_{t+h-\tau} f(u(\tau)) d \tau .
$$


Remark 4.7. In this paper we do not consider the question of uniqueness of solutions and indeed, Definition 4.1 may be too relaxed to ascertain it. In Kru17 it is shown that the semigroup solution $P_{t} u_{0}$ is in fact the unique solution to the linear problem under additional continuity-in-time assumptions. In the remainder we only work with the Picard solutions to problem (11), which are well-defined.

\section{REgularity OF SOLUtions}

5.1. Moment estimates. In the first part of this section we reproduce the second moment estimates presented in Ros68. We are only able to do this while assuming higher regularity of the initial condition, namely $u_{0} \in \mathrm{Iso}_{2}^{1}$. Despite this limitation, the result is interesting because of an elegant identity described in Remark 5.5. In the sequel we obtain weaker (but sufficient) estimates for all moments in a more general setting.

Lemma 5.1. Suppose $u$ is the Picard solution to (11). Let $g: \mathbb{R} \rightarrow \mathbb{R}$ be a measurable function such that $g(u(t)) \in \mathrm{Iso}_{2}$ for some $t \geq 0$. Then for every $h>0$ we have

$$
E u(t+h) g(u(t))=E P_{h} u(t) g(u(t))+h R(h),
$$

where $R:[0, \infty) \rightarrow \mathbb{R}$ is a function such that $\lim _{h \rightarrow 0} R(h)=0$.

Proof. From Corollary 4.6 we have

$$
u(t+h)=P_{h} u(t)+\int_{t}^{t+h} \nabla_{z} P_{t+h-\tau} f(u(\tau)) d \tau .
$$

We define $R(h)=h^{-1} E \int_{t}^{t+h} \nabla_{z} P_{t+h-\tau} f(u(\tau)) d \tau g(u(t))$. By the Lebesgue differentiation theorem and Lemma 2.11 we obtain

$$
\lim _{h \rightarrow 0} E \frac{1}{h} \int_{t}^{t+h} \nabla_{z} P_{t+h-\tau} f(u(\tau)) d \tau g(u(t))=E \nabla_{z} f(u(t)) g(u(t))=0 .
$$

Corollary 5.2. Suppose $u_{0} \in \mathrm{IsO}_{2}$ and $u$ is the Picard solution to (11). Then

$$
\lim _{h \rightarrow 0} E \frac{(u(t+h)-u(t))^{2}}{h}=0 .
$$

Proof. Notice that if $a=b+c$ then $a^{2}=b^{2}+c(b+a)$. It follows from Corollary 4.6 that

$$
u(t+h)^{2}=\left(P_{h} u(t)\right)^{2}+\left(\int_{t}^{t+h} \nabla_{z} P_{t+h-\tau} f(u(\tau)) d \tau\right)\left(P_{h} u(t)+u(t+h)\right) .
$$

Then by Lemma 5.1 we get

$$
\begin{aligned}
& E(u(t+h)-u(t))^{2}=E u(t+h)^{2}-2 E u(t+h) u(t)+E u(t)^{2} \\
& =E\left(P_{h} u(t)\right)^{2}+E\left(\int_{t}^{t+h} \nabla_{z} P_{t+h-\tau} f(u(\tau)) d \tau\right)\left(P_{h} u(t)+u(t+h)\right) \\
& -2 E P_{h} u(t) u(t)+E u(t)^{2}+h R(h),
\end{aligned}
$$

where $\lim _{h \rightarrow 0} R(h)=0$. This, combined with the Lebesgue differentiation theorem, implies

$$
\lim _{h \rightarrow 0} E \frac{(u(t+h)-u(t))^{2}}{h}=\lim _{h \rightarrow 0} E \frac{\left(P_{h} u(t)-u(t)\right)^{2}}{h}=0 .
$$


Lemma 5.3. If $u$ is the Picard solution to problem (11) with $u_{0} \in \mathrm{Iso}_{2}^{1}$ then $u \in C\left([0, \infty), \operatorname{Iso}_{2}^{1}\right)$.

Proof. Let $u_{n}$ be the sequence of Picard iterations as defined in (14) and let $K_{0}$ be such as in Theorem 4.4, i.e.

$$
c_{s} L K_{0}^{-1+\frac{1}{2 s}} \Gamma\left(1-\frac{1}{2 s}\right)<1
$$

where $c_{s}$ is a known constant.

Let us proceed by induction. We have

$$
\left\|u_{1}(t)\right\|_{1,2}^{2}=\int\left(1+|\xi|^{2 s}\right) e^{-2 t|\xi|^{2 s}} \sigma(d \xi) 3 \leq\left\|u_{0}\right\|_{1,2}^{2}
$$

hence $u_{1} \in C\left([0, \infty), \operatorname{Iso}_{2}^{1}\right)$.

Suppose $u_{n} \in C\left([0, \infty), \mathrm{Iso}_{2}^{1}\right)$ and $\sup _{t>0} e^{-t K_{0}}\left\|u_{n}(t)\right\|_{1,2} \leq n\left\|u_{0}\right\|_{1,2}$. By Lemma 3.12 and Corollary 2.15 for every $t>0$ we have

$$
\left\|\nabla_{z} P_{t} f\left(u_{n}(\tau)\right)\right\|_{1,2} \leq c_{s} t^{-1 / 2 s}\left\|f\left(u_{n}(\tau)\right)\right\|_{1,2} \leq c_{s} L t^{-1 / 2 s}\left\|u_{n}(\tau)\right\|_{1,2} .
$$

Therefore

$$
\begin{aligned}
\left\|u_{n+1}(t)\right\|_{1,2} \leq\left\|u_{1}(t)\right\|_{1,2} & +\int_{0}^{t}\left\|\nabla_{z} P_{t-\tau} f\left(u_{n}(\tau)\right)\right\|_{1,2} d \tau \\
& \leq\left\|u_{1}(t)\right\|_{1,2}+c_{s} L \int_{0}^{t}(t-\tau)^{-1 / 2 s}\left\|u_{n}(\tau)\right\|_{1,2} d \tau .
\end{aligned}
$$

Using estimates (12), (13), (15) and the induction hypothesis, we thus obtain

$$
\begin{aligned}
\sup _{t \geq 0} e^{-t K_{0}} & \left\|u_{n+1}(t)\right\|_{1,2} \\
& \leq\left\|u_{1}(t)\right\|_{1,2}+n c_{s} L K_{0}^{-1+\frac{1}{2 s}} \Gamma\left(1-\frac{1}{2 s}\right)\left\|u_{0}\right\|_{1,2} \leq(n+1)\left\|u_{0}\right\|_{1,2} .
\end{aligned}
$$

It follows that $u_{n+1} \in C\left([0, \infty), \operatorname{Iso}_{2}^{1}\right)$.

Moreover, we obtain

$$
\begin{aligned}
\sup _{t \geq 0} e^{-t K_{0}} \| u_{n}(t)- & u_{m}(t) \|_{1,2} \\
& \leq c_{s} L K^{-1+\frac{1}{2 s}} \Gamma\left(1-\frac{1}{2 s}\right) \sup _{t \geq 0} e^{-t K_{0}}\left\|u_{n-1}-u_{m-1}\right\|_{1,2}
\end{aligned}
$$

and because of (15) it follows that $\left\{u_{n}\right\}$ is a Cauchy sequence in $C\left([0, T], \mathrm{Iso}_{2}^{1}\right)$ for every $T>0$ and $u \in C\left([0, \infty)\right.$, $\left.\operatorname{Iso}_{2}^{1}\right)$.

Theorem 5.4. If $u$ is the Picard solution to (11) with $u_{0} \in \mathrm{Iso}_{2}^{1}$ then

$$
\partial_{t} E u(t)^{2}=-2 E\left((-\Delta)^{s / 2} u(t)\right)^{2} .
$$

Proof. From Lemma 5.1 we have

$$
E \frac{u(t+h)-u(t)}{h} u(t)=E \frac{P_{h} u(t)-u(t)}{h} u(t)+R(h),
$$

where $\lim _{h \rightarrow 0} R(h)=0$. Notice that by Lemma 5.3 and Proposition 2.13 we have $u(t) \in \mathrm{Iso}_{2}^{1} \subset \mathrm{Iso}_{2}^{s}$ for $s \leq 1$. Therefore on the right-hand side of equality (16) by Proposition 3.6 we get

$$
\lim _{h \rightarrow 0}\left(E \frac{P_{h} u(t)-u(t)}{h} u(t)+R(h)\right)=-E\left((-\Delta)^{s / 2} u(t)\right)^{2} .
$$


This ensures the existence of the limit on the left-hand side and by Corollary 5.2 we obtain

$$
\begin{aligned}
& \lim _{h \rightarrow 0} \frac{E(u(t+h)-u(t))}{h} u(t)=\frac{1}{2} \lim _{h \rightarrow 0} \frac{E(u(t+h)-u(t))}{h}(u(t)+u(t+h)) \\
&=\frac{1}{2} \lim _{h \rightarrow 0} \frac{E\left(u(t+h)^{2}-u(t)^{2}\right)}{h}=\frac{1}{2} \partial_{t} E u(t)^{2} .
\end{aligned}
$$

Remark 5.5. For the solution $u$ as in Theorem 5.4 we define the covariance functional $B(t, y)=E u(t, x) u(t, x+y)$. Let us notice that $\partial_{t} B(t, 0)=\partial_{t} E u(t, x)^{2}$ and according to Definition 3.1 we have

$$
\begin{gathered}
\left.\left(-\Delta_{y}^{s}\right) B(t, y)\right|_{y=0}=\int_{\mathbb{R}^{d}}|\zeta|^{2 s} \int_{\mathbb{R}^{d}} e^{-i z \cdot \zeta} E u(t, x) u(t, x+z) d z d \zeta \\
=\int_{\mathbb{R}^{d}}|\zeta|^{2 s} \int_{\mathbb{R}^{d}} e^{-i z \cdot \zeta} \int_{\mathbb{R}^{d}} e^{i z \cdot \xi} d \sigma_{t}(\xi) d z d \zeta=\int_{\mathbb{R}^{d}}|\zeta|^{2 s} \sigma_{t}(d \zeta) \\
=E\left((-\Delta)^{s / 2} u(t)\right)^{2} .
\end{gathered}
$$

Therefore we may write the result of Theorem 5.4 as

$$
\partial_{t} B(t, 0)=-\left.2\left(-\Delta_{y}^{s}\right) B(t, y)\right|_{y=0} .
$$

Note that this property of the functional $B(t, y)$ holds only at $y=0$.

5.2. Higher moments estimates. In order to prove the estimates similar to those obtained (for the second moment) in Theorem 5.4 for moments $p>2$, we need to fall back to the classical theory applied pathwise because of issues with regularity. As indicated in Remark 3.9, this is only possible in the space $\mathrm{Iso}_{\infty}$. Accordingly, we will work with cut-off initial data first and then reach the general case by approximation.

Lemma 5.6. If $u_{0} \in \mathrm{Iso}_{\infty}$ and $f \in C^{\infty}(\mathbb{R})$ then for every $\omega \in \Omega$ there exists a unique function $u^{\omega}(t, x) \in C^{\infty}\left((0, \infty) \times \mathbb{R}^{d}\right) \cap L^{\infty}\left([0, \infty) \times \mathbb{R}^{d}, d x\right)$ which is a (classical) solution to

$$
\left\{\begin{array}{lr}
\partial_{t} u+(-\Delta)^{s} u=\nabla_{z} f(u) & \text { on }[0, \infty) \times \mathbb{R}^{d}, \\
u^{\omega}(0)=u_{0}(x, \omega) & \text { a.e. on } \mathbb{R}^{d} .
\end{array}\right.
$$

Proof. We know from Proposition 3.8 that $u_{0}(x, \omega) \in L^{\infty}\left(\mathbb{R}^{d}, d x\right)$ for every $\omega \in \Omega$. Because we assume $f \in C^{\infty}(\mathbb{R})$, it follows from [DGV03, Theorem 1.1] that there exists a unique solution $u^{\omega}$ to problem (17), such that $u^{\omega} \in C^{\infty}\left((0, \infty) \times \mathbb{R}^{d}\right) \cap$ $L^{\infty}\left([0, \infty) \times \mathbb{R}^{d}, d x\right)$.

Lemma 5.7. Let $f$ be Lipschitz. Suppose $u$ is the Picard solution to (11) with $u_{0} \in \mathrm{Iso}_{\infty}$ and $f \in C^{\infty}(\mathbb{R})$. Let $u^{\omega}$ be defined as in Lemma 5.6 for every $\omega \in \Omega$. Then $u^{\omega}(t, x)=u(t, x, \omega)$ for almost all $\omega \in \Omega$.

Proof. Recall we denote by

$$
\mathcal{B}_{K, L^{\infty}\left(\mathbb{R}^{d}, d x\right)}=\left\{u \in C\left([0, \infty), L^{\infty}\left(\mathbb{R}^{d}, d x\right)\right): \sup _{t \geq 0} e^{-t K}\|u(t)\|_{L^{\infty}\left(\mathbb{R}^{d} . d x\right)}\right\}
$$

the Banach space of continuous functions endowed with an appropriate Bielecki norm.

Let us proceed by induction. It follows from Proposition 3.8 that

$$
u_{1}^{\omega}(t, x)=u_{1}(t, x, \omega)=P_{t} u_{0}(x, \omega)
$$

for almost every $\omega \in \Omega$, as well as that for every $\omega \in \Omega$ and every $K>0$ we have $u_{1}^{\omega} \in \mathcal{B}_{K, L^{\infty}(d x)}$. Let $K_{0}$ be such as in Theorem 4.4 the sequence $u_{n}$ be defined as 
in (14) and $u_{n}^{\omega}$ be analogous sequences of (non-random) Picard iterations starting with $u_{1}^{\omega}$ for every $\omega \in \Omega$.

Suppose we have $u_{n}(t, x, \omega)=u_{n}^{\omega}(t, x)$ for almost every $\omega \in \Omega$ and $u_{n}^{\omega}(t, x) \in$ $\mathcal{B}_{K, L^{\infty}(d x)}$ for every $\omega \in \Omega$ and every $K \geq K_{0}$. Then because of the Hille theorem (as in the proof of Proposition 3.8) we get

$$
\left(\int_{0}^{t} \nabla_{z} P_{t-\tau} f\left(u_{n}(\tau, x)\right) d \tau\right)(\omega)=\int_{0}^{t} \nabla_{z} P_{t-\tau} f\left(u_{n}^{\omega}(\tau, x)\right) d \tau .
$$

It follows that $u_{n+1}(t, x, \omega)=u_{n+1}^{\omega}(t, x)$ and moreover

$$
\begin{aligned}
\left\|u_{n+1}^{\omega}(t)\right\|_{L^{\infty}(d x)} & \leq\left\|u_{1}^{\omega}(0)\right\|_{L^{\infty}(d x)}+\int_{0}^{t}\left\|\nabla_{z} P_{t-\tau} f\left(u_{n}^{\omega}(\tau)\right)\right\|_{L^{\infty}(d x)} d \tau \\
& \leq\left\|u_{1}^{\omega}(0)\right\|_{L^{\infty}(d x)}+c_{s} L \int_{0}^{t}(t-\tau)^{-1 / 2 s}\left\|u_{n}^{\omega}(\tau)\right\|_{L^{\infty}(d x)} d \tau .
\end{aligned}
$$

This gives us an estimate

$$
\begin{aligned}
& \left\|u_{n+1}^{\omega}\right\| \|_{K, L^{\infty}\left(\mathbb{R}^{d}, d x\right)} \\
& \quad \leq\left\|u_{1}^{\omega}\right\|_{K, L^{\infty}\left(\mathbb{R}^{d}, d x\right)}+c_{s} L K^{-1+\frac{1}{2 s}} \Gamma\left(1-\frac{1}{2 s}\right)\left\|u_{n}^{\omega}\right\|_{K, L^{\infty}\left(\mathbb{R}^{d}, d x\right)},
\end{aligned}
$$

which, because we have $K \geq K_{0}$, asserts that $\left\{u_{n}\right\} \subset \mathcal{B}_{K_{0}, L^{\infty}(d x)}$.

Consequently $u^{\omega}(t, x)=u(t, x, \omega)$, for almost every $\omega \in \Omega$, since both solutions are defined as limits of Picard iterations.

Proposition 5.8. Let $w \in \mathrm{Iso}_{\infty}, \theta: \mathbb{R}^{d} \rightarrow \mathbb{R}$ be a measurable function such that $|\theta|=1$ and $a+b=2$. Then

$$
E P_{h} \theta|w|^{a} \theta|w|^{b} \leq a b E P_{h}|w||w|
$$

Proof. Let us notice that, because $\theta(x)|w(x)|{ }^{a} \theta(x)|w(x)|^{b}=|w(x)|^{2}$ and $p_{h}(x-y)=$ $p_{h}(y-x)$, we have

$$
\begin{aligned}
& E|w|^{2}-E P_{h} \theta|w|^{a} \theta|w|^{b}=E|w|^{2}-\int_{\mathbb{R}^{d}} p_{h}(x-y) \theta(y)|w(y)|^{a} \theta(x)|w(x)|^{b} d y \\
& =\frac{1}{2} \int_{\mathbb{R}^{d}} p_{h}(x-y) E\left(\theta(y)|w(y)|^{a}-\theta(x)|w(x)|^{a}\right)\left(\theta(y)|w(y)|^{b}-\theta(x)|w(x)|^{b}\right) d y .
\end{aligned}
$$

Similarly,

$$
\begin{aligned}
& E P_{h}|w||w|=\int_{\mathbb{R}^{d}} p_{h}(x-y) E|w(y)||w(x)| d y \\
&=E|w|^{2}-\frac{1}{2} \int_{\mathbb{R}^{d}} p_{h}(x-y) E(|w(y)|-|w(x)|)^{2} d y .
\end{aligned}
$$

We may now use the following inequality (see [VSCC92, Lemma II.5.5]

$$
\left(\theta_{1} x^{a}-\theta_{2} y^{a}\right)\left(\theta_{1} x^{b}-\theta_{2} y^{b}\right) \geq a b(x-y)^{2}, \text { when } a+b=2 \text { and }\left|\theta_{1}\right|=\left|\theta_{2}\right|=1
$$

to obtain the desired result.

Lemma 5.9. If $u$ is the Picard solution to (11) with $u_{0} \in \operatorname{Iso}_{\infty}$ and $f \in C^{\infty}(\mathbb{R})$ then

$$
\partial_{t} E|u|^{p} \leq-\left.\left.4 \frac{p-1}{p} E\left|(-\Delta)^{s / 2}\right| u\right|^{p}\right|^{2}
$$

for every $p \geq 2$. 
Proof. From Lemma 5.1 we have

$$
\begin{aligned}
& E \frac{u(t+h)-u(t)}{h}|u|^{p-1}(t) \operatorname{sgn} u(t) \\
& \quad=E \frac{P_{h} u(t)-u(t)}{h}|u|^{p-1}(t) \operatorname{sgn} u(t)+R(h),
\end{aligned}
$$

where $\lim _{h \rightarrow 0} R(h)=0$. Let $w=|u|^{\frac{p-2}{2}} u$. Then

$$
P_{h} u\left(|u|^{p-1} \operatorname{sgn} u\right)=P_{h}\left(|w|^{2 / p} \operatorname{sgn} w\right)\left(|w|^{\frac{2(p-1)}{p}} \operatorname{sgn} w\right)
$$

and it follows from Proposition 5.8 that

$$
E\left(P_{h} u(t)-u(t)\right)|u|^{p-1}(t) \operatorname{sgn} u(t) \leq 4 \frac{p-1}{p^{2}} E\left(P_{h}|w|-|w|\right)|w| .
$$

Because $u(t) \in \mathrm{Iso}_{2}^{1} \cap \mathrm{Iso}_{\infty}$ for $t>0$ then by Corollary 2.15 $w \in \mathrm{Iso}_{2}^{1} \subset \mathrm{Iso}_{2}^{s}$ and we have

$$
\lim _{h \rightarrow 0} E \frac{\left(P_{h}|w|-|w|\right)|w|}{h}=-E\left|(-\Delta)^{s / 2}\right| w||^{2} .
$$

On the other hand, it follows from Lemmas 5.6 and 5.7 that $u(t, x, \omega)=u^{\omega}(t, x)$ for almost every $\omega \in \Omega$ and $u^{\omega} \in C^{\infty}\left((0, \infty) \times \mathbb{R}^{d}\right)$, therefore

$$
\lim _{h \rightarrow 0} E \frac{u(t+h)-u(t)}{h}|u|^{p-1}(t) \operatorname{sgn} u(t)=\frac{1}{p} \partial_{t} E|u|^{p},
$$

which yields the result.

Theorem 5.10. If $u$ is the Picard solution to (11) with $u_{0} \in \operatorname{Iso}_{p}$ and $f \in C^{\infty}(\mathbb{R})$ then

$$
E|u(t)|^{p} \leq E\left|u_{0}\right|^{p}
$$

for every $2 \leq p \leq \infty$ and every $t \geq 0$.

Proof. We consider the sequence $u_{0}^{n}=h_{n}\left(u_{0}\right)$, where $h_{n}$ are cut-off functions $h_{n}(x)=\min \{|x|, n\} \operatorname{sgn}(x)$ and the sequence $u_{n}$ of solutions to problems

$$
\left\{\begin{array}{lr}
\partial_{t} u+(-\Delta)^{s} u=\nabla_{z} f(u) & \text { on }[0, \infty) \times \mathbb{R}^{d}, \\
u(0) \stackrel{d}{=} u_{0}^{n} & \text { on } \mathbb{R}^{d} .
\end{array}\right.
$$

as constructed in Theorem 4.4. Then $u_{0}^{n} \in \mathrm{Iso}_{\infty}$ and it follows from Lemma 5.9. that

$$
E\left|u_{n}(t)\right|^{p} \leq E\left|u_{0}^{n}\right|^{p}
$$

for every $n \geq 1, t \geq 0$ and $p \geq 2$. By Lemma 4.3 we know that

$$
\lim _{n \rightarrow \infty}\left\|u_{n}-u\right\|_{p}=0
$$

and the result follows.

\section{Nonlinearity with Polynomial Growth}

We consider the following initial value problem

$$
\left\{\begin{array}{lr}
\partial_{t} u+(-\Delta)^{s} u=\nabla_{z} f(u) & \text { on }[0, \infty) \times \mathbb{R}^{d}, \\
u(0) \stackrel{d}{=} u_{0} & \text { on } \mathbb{R}^{d} .
\end{array}\right.
$$

Here we assume $s \in\left(\frac{1}{2}, 1\right], u_{0} \in \bigcap_{p \geq 2} \operatorname{Iso}_{p}$ and $f \in C^{\infty}(\mathbb{R}), f(0)=0$ and $\mid f(u)-$ $f(v)|\leq C| u-v \mid\left(|u|^{q}+|v|^{q}\right)$ for some constants $C>0$ and $q \geq 1$. By referring to problem (18) we also quietly include these assumptions. 


\subsection{Existence of solutions.}

Definition 6.1. Given $u \in \bigcap_{p \geq 2} \mathrm{~J}^{-I_{s o}, p}$ for some $K \geq 0$, we define the following nonlinear operator

$$
F(u)(t)=P_{t} u(0)+\int_{0}^{t} \nabla_{z} P_{t-\tau} f(u(\tau)) d \tau .
$$

Let $u_{0} \in \bigcap_{p \geq 2} \operatorname{Iso}_{p}$. For $K \geq 0$ we say that $u \in \bigcap_{p \geq 1}$ J-Iso $K, p_{\text {is a solution to }}$ is problem (18) if $F(u)=u$ and $u_{0} \stackrel{d}{=} u(0)$.

In order to prove the existence of solutions we need to consider a sequence of approximations defined in the following way. Take $u_{0}^{n}=h_{n}\left(u_{0}\right)$, where $h_{n}$ are cutoff functions $h_{n}(x)=\min \{|x|, n\} \operatorname{sgn}(x)$ and define the sequence of Picard solutions $u^{n}$ to problems

$$
\left\{\begin{array}{lr}
\partial_{t} u^{n}+(-\Delta)^{s} u^{n}=\nabla_{z} f\left(h_{n}\left(u^{n}\right)\right) & \text { on }[0, \infty) \times \mathbb{R}^{d}, \\
u^{n}(0) \stackrel{d}{=} u_{0}^{n} & \text { on } \mathbb{R}^{d} .
\end{array}\right.
$$

The function $f\left(h_{n}(x)\right)$ is Lipschitz and thus, because of Theorem 4.4, such a sequence exists. We call it the sequence of approximative solutions throughout this section.

Remark 6.2. For every $n$, the solution $u^{n}$ of problem (19) is by definition a fixed point of the operator

$$
F_{n}(u)(t)=P_{t} u(0)+\int_{0}^{t} \nabla_{z} P_{t-\tau} f\left(h_{n}(u(t))\right) d \tau .
$$

In the following proposition we show that the approximative solutions $u^{n}$ are not only solutions to problems (19) but also solutions to problem (18), with the cut-off initial conditions $u_{0}^{n}$, respectively. In other words, it turns out we do not need to cut-off the function $f$, once we have constructed solutions $u^{n}$ as limits of Picard iterations linked to problems (19).

Proposition 6.3. Let $u^{n}$ be the sequence of approximative solutions. Then $u^{n}$ are fixed points of the operator $F$.

Proof. It follows from Theorem 5.10 that $\left\|u^{n}(t)\right\|_{\infty} \leq\left\|u_{0}^{n}\right\|_{\infty} \leq n$ for every $n \geq 1$ and $t \geq 0$. Therefore $f\left(h_{n}\left(u^{n}(t)\right)=f\left(u^{n}(t)\right)\right.$. The Picard solution $u^{n}$ is a fixed point of the operator $F_{n}$ given by (20) and hence $u^{n}=F_{n}\left(u^{n}\right)=F\left(u^{n}\right)$.

Lemma 6.4. The sequence of approximative solutions is convergent in $\mathrm{J}_{-} \mathrm{Iso}_{K, p}$ for every $p \geq 2$ and every $K>0$.

Proof. It follows from Lemma 5.7 that $u_{n}(\omega)$ are also classical solutions for almost every $\omega \in \Omega$, therefore we may write

$$
\partial_{t}\left(u_{n}-u_{m}\right)=-(-\Delta)^{s}\left(u_{n}-u_{m}\right)+\nabla_{z}\left(f\left(u_{n}\right)-f\left(u_{m}\right)\right) .
$$

Let $g(x)=|x|^{p-1} \operatorname{sgn}(x)$. Because

$$
f\left(u_{n}\right)-f\left(u_{m}\right)=\bar{f}\left(u_{n}, u_{m}\right) \sim \bar{g}\left(u_{n}, u_{m}\right)=g\left(u_{n}-u_{m}\right),
$$

it follows from Lemma 2.11 that

$$
E \nabla_{z}\left(f\left(u_{n}\right)-f\left(u_{m}\right)\right)\left|u_{n}-u_{m}\right|^{p-1} \operatorname{sgn}\left(u_{n}-u_{m}\right)=0,
$$

hence

$$
E \partial_{t}\left(u_{n}-u_{m}\right) g\left(u_{n}-u_{m}\right)=-E(-\Delta)^{s}\left(u_{n}-u_{m}\right) g\left(u_{n}-u_{m}\right) .
$$

Notice that $g$ is a convex function, therefore

$$
(-\Delta)^{s} g\left(u_{n}(\omega)-u_{m}(\omega)\right) \leq(-\Delta)^{s}\left(u_{n}(\omega)-u_{m}(\omega)\right) g\left(u_{n}(\omega)-u_{m}(\omega)\right)
$$


for almost every $\omega \in \Omega$ (see [CS17, Theorem 1.1]), and because of the regularity of classical solutions (see Theorem [5.6) and Remark 3.3] we have

$$
\partial_{t} E\left|u_{n}-u_{m}\right|^{p}=-E(-\Delta)^{s}\left(u_{n}-u_{m}\right) g\left(u_{n}-u_{m}\right) \leq-E(-\Delta)^{s} g\left(u_{n}-u_{m}\right)=0 .
$$

It follows that

$$
E\left|u_{n}(t)-u_{m}(t)\right|^{p} \leq E\left|u_{0}^{n}-u_{0}^{m}\right|^{p}
$$

for every $t \geq 0$. However,

$$
\lim _{n, m \rightarrow \infty} E\left|u_{0}^{n}-u_{0}^{m}\right|=0,
$$

thus

$$
\lim _{n, m \rightarrow \infty} \sup _{t \geq 0} E\left|u_{n}(t)-u_{m}(t)\right|^{p}=0,
$$

which shows that $u^{n}$ is a Cauchy sequence, and therefore converges, in $\mathrm{J}_{-} \mathrm{Iso}_{K, p}$.

Theorem 6.5. There exists a solution $u$ to problem (18) such that $E|u(t)|^{p} \leq$ $E\left|u_{0}\right|^{p}$ for every $t \geq 0$ and $p \geq 2$.

Proof. Let $u$ be the limit of the approximative solutions $u^{n}$ given by Lemma 6.4 The estimates

$$
E|u(t)|^{p} \leq E\left|u_{0}\right|^{p}
$$

follow from the analogous properties of the approximative solutions given in Theorem 5.10. Consider

$$
F(u)=P_{t} u(0)+\int_{0}^{t} \nabla_{z} P_{t-\tau} f(u(\tau)) d \tau .
$$

By Lemma 3.13 we have

$$
\begin{aligned}
\| u^{n}(t)- & F(u)(t) \|_{2} \\
& \leq\left\|P_{t}\left(u_{0}^{n}-u_{0}\right)\right\|_{2}+c_{s} \int_{0}^{t}(t-\tau)^{-\frac{1}{2 s}}\left\|f\left(u^{n}(\tau)\right)-f(u(\tau))\right\|_{2} d \tau .
\end{aligned}
$$

Because we assume $|f(x)-f(y)| \leq C|x-y|\left(|x|^{q}+|y|^{q}\right)$, then

$$
\begin{aligned}
& \left\|u^{n}(t)-F(u)(t)\right\|_{2}-\left\|P_{t}\left(u_{0}^{n}-u_{0}\right)\right\|_{2} \\
& \quad \leq c_{s} C \int_{0}^{t}(t-\tau)^{-\frac{1}{2 s}}\left\|u^{n}(\tau)-u(\tau)\right\|_{2}\left(\left\|u^{n}(\tau)\right\|_{2}^{q}+\|u(\tau)\|_{2}^{q}\right) d \tau .
\end{aligned}
$$

By Theorem 5.10 and estimate (21) we get

$$
\begin{aligned}
\int_{0}^{t}(t-\tau)^{-\frac{1}{2 s}}\left\|u^{n}(\tau)-u(\tau)\right\|_{2} & \left(\left\|u^{n}(\tau)\right\|_{2}^{q}+\|u(\tau)\|_{2}^{q}\right) d \tau \\
& \leq 2\left\|u_{0}\right\|_{2}^{q} \int_{0}^{t}(t-\tau)^{-\frac{1}{2 s}}\left\|u^{n}(\tau)-u(\tau)\right\|_{2} d \tau .
\end{aligned}
$$

For every $K>0$ (cf. estimates (12), (13)) we have

$$
\begin{aligned}
& \sup _{t \geq 0} e^{-t K} \int_{0}^{t}(t-\tau)^{-\frac{1}{2 s}}\left\|u^{n}(\tau)-u(\tau)\right\|_{2} d \tau \\
& \leq\left(\sup _{0<\tau<t} e^{-\tau K}\left\|u^{n}(\tau)-u(\tau)\right\|_{2}\right) \int_{0}^{t}(t-\tau)^{-1 / 2 s} e^{-(t-\tau) K} d \tau \\
& \quad \leq K^{-1+\frac{1}{2 s}} \Gamma\left(1-\frac{1}{2 s}\right)\left\|u^{n}-u\right\|_{K, 2} .
\end{aligned}
$$

Finally we obtain

$$
\left\|u^{n}-F(u)\right\|_{K, 2} \leq c_{s} C K^{-1+\frac{1}{2 s}} \Gamma\left(1-\frac{1}{2 s}\right)\left\|u^{n}-u\right\|_{K, 2}
$$


and by Lemma 6.4

$$
\lim _{n \rightarrow \infty}\left\|u^{n}-F(u)\right\|_{K, 2}=0 .
$$

This of course means that $u=F(u)$ (Lemma 6.4 again) and $u$ is a solution.

Remark 6.6. As in Remark 4.7, the solution we have constructed in this section may not be unique in the context of Definition 6.1.

\section{ACKNOWLEDGEMENTS}

The author wishes to thank Grzegorz Karch for his invaluable help in preparing this paper. This work was partially supported by the Polish NCN grant No. 2016/23/B/ST1/00434.

\section{REFERENCES}

[AL01] V. V. Anh and N. N. Leonenko, Spectral analysis of fractional kinetic equations with random data, J. Statist. Phys. 104 (2001), no. 5-6, 1349-1387.

[AL02] - Renormalization and homogenization of fractional diffusion equations with random data, Probab. Theory Related Fields 124 (2002), no. 3, 381-408.

[AMS94] S. Albeverio, S. A. Molchanov, and D. Surgailis, Stratified structure of the Universe and Burgers' equation - a probabilistic approach, Probab. Theory Related Fields 100 (1994), no. 4, 457-484.

[Bak01] Yu. Yu. Bakhtin, A functional central limit theorem for transformed solutions of the multidimensional Burgers equation with random initial data, Teor. Veroyatnost. i Primenen. 46 (2001), no. 3, 427-448. MR 1978661

[Bil95] P. Billingsley, Probability and measure, third ed., Wiley Series in Probability and Mathematical Statistics, John Wiley \& Sons, Inc., New York, 1995, A WileyInterscience Publication.

[BKW99] P. Biler, G. Karch, and W. A. Woyczyński, Asymptotics for multifractal conservation laws, Studia Math. 135 (1999), no. 3, 231-252.

[BKW01a] _ Asymptotics for conservation laws involving Lévy diffusion generators, Studia Math. 148 (2001), no. 2, 171-192.

[BKW01b] — Critical nonlinearity exponent and self-similar asymptotics for Lévy conservation laws, Ann. Inst. H. Poincaré Anal. Non Linéaire 18 (2001), no. 5, 613-637.

[BPSV14] B. Barrios, I. Peral, F. Soria, and E. Valdinoci, A Widder's type theorem for the heat equation with nonlocal diffusion, Arch. Ration. Mech. Anal. 213 (2014), no. 2, 629-650.

[CS17] L. A. Caffarelli and Y. Sire, On some pointwise inequalities involving nonlocal operators, Harmonic analysis, partial differential equations and applications, Appl. Numer. Harmon. Anal., Birkhäuser/Springer, Cham, 2017, pp. 1-18.

[DGV03] J. Droniou, T. Gallouet, and J. Vovelle, Global solution and smoothing effect for a non-local regularization of a hyperbolic equation, J. Evol. Equ. 3 (2003), no. 3, 499521, Dedicated to Philippe Bénilan.

[GS69] I. I. Gikhman and A. V. Skorokhod, Introduction to the theory of random processes, Translated from the Russian by Scripta Technica, Inc, W. B. Saunders Co., Philadelphia, Pa., 1969

[GV64] I. M. Gel'fand and N. Ya. Vilenkin, Generalized functions. Vol. 4: Applications of harmonic analysis, Translated by Amiel Feinstein, Academic Press, New York, 1964.

[IL89] A. V. Ivanov and N. N. Leonenko, Statistical analysis of random fields, Mathematics and its Applications (Soviet Series), vol. 28, Kluwer Academic Publishers Group, Dordrecht, 1989, With a preface by A. V. Skorokhod, Translated from the Russian by A. I. Kochubinskiı̌.

[IS17] L. Ignat and D. Stan, Asymptotic behaviour for fractional diffusion-convection equations, ArXiv e-prints (2017).

[JW01] J. A. Mann Jr. and W. A. Woyczyński, Growing fractal interfaces in the presence of self-similar hopping surface diffusion, Physica A: Statistical Mechanics and its Applications 291 (2001), no. 1-4, 159-183.

[Kru17] M. Krupski, Scaling limits of solutions of linear evolution equations with random initial conditions, Stoch. Dyn. 17 (2017), no. 3, 1750019, 24.

[KW08] G. Karch and W. A. Woyczyński, Fractal Hamilton-Jacobi-KPZ equations, Trans. Amer. Math. Soc. 360 (2008), no. 5, 2423-2442. 
[Leo99] N. N. Leonenko, Limit theorems for random fields with singular spectrum, Mathematics and its Applications, vol. 465, Kluwer Academic Publishers, Dordrecht, 1999.

[LS96] V. A. Liskevich and Yu. A. Semenov, Some problems on Markov semigroups, Schrödinger operators, Markov semigroups, wavelet analysis, operator algebras, Math. Top., vol. 11, Akademie Verlag, Berlin, 1996, pp. 163-217.

[LW98] N. N. Leonenko and W. A. Woyczyński, Exact parabolic asymptotics for singular $n-D$ Burgers' random fields: Gaussian approximation, Stochastic Process. Appl. 76 (1998), no. 2, 141-165.

[Rao12] M. M. Rao, Random and vector measures, Series on Multivariate Analysis, vol. 9, World Scientific Publishing Co. Pte. Ltd., Hackensack, NJ, 2012.

[Ros68] M. Rosenblatt, Remarks on the Burgers equation, J. Mathematical Phys. 9 (1968), $1129-1136$.

[VSCC92] N. Th. Varopoulos, L. Saloff-Coste, and T. Coulhon, Analysis and geometry on groups, Cambridge Tracts in Mathematics, vol. 100, Cambridge University Press, Cambridge, 1992.

[Woy98] W. A. Woyczyński, Burgers-KPZ turbulence, Lecture Notes in Mathematics, vol. 1700, Springer-Verlag, Berlin, 1998, Göttingen lectures. 